\title{
Management of acute pancreatitis (AP) - Polish Pancreatic Club recommendations
}

\author{
Mariusz Rosołowski ${ }^{1}$, Michał Lipiński ${ }^{2}$, Marek Dobosz ${ }^{3,4}$, Marek Durlik ${ }^{5,6}$, Stanisław Głuszek ${ }^{7,8}$, \\ Katarzyna Kuśnierz ${ }^{9}$, Paweł Lampe ${ }^{9}$, Ewa Małecka-Panas ${ }^{10}$, Ewa Nowakowska-Duława ${ }^{11}$, \\ Magdalena Nowak-Niezgoda ${ }^{6}$, Barbara Radomańska ${ }^{12}$, Renata Talar-Wojnarowska ${ }^{10}$, \\ Urszula Wereszczyńska-Siemiątkowska ${ }^{13}$, Grażyna Rydzewska ${ }^{2,14}$
}

${ }^{1}$ Department of Gastroenterology and Internal Medicine, Medical University of Bialystok, Bialystok, Poland 2Department of Internal Medicine and Gastroenterology with Inflammatory Bowel Disease Subdivision, Central Clinical Hospital of the Ministry of the Interior, Warsaw, Poland ${ }^{3}$ Department of Nursing, Medical University of Gdansk, Gdansk, Poland ${ }^{4}$ Department of General and Gastroenterological Surgery and Nutrition, Copernicus, Gdansk, Poland ${ }^{5}$ Mossakowski Medical Research Centre, Polish Academy of Sciences, Warsaw, Poland ${ }^{6}$ Clinical Department of Gastroenterological Surgery and Transplantation, Central Clinical Hospital of the Ministry of the Interior, Warsaw, Poland ${ }^{7}$ Department of Surgery and Surgical Nursing, Institute of Medical Sciences, Faculty of Medicine and Health Science, Jan Kochanowski University, Kielce, Poland ${ }^{8}$ Clinical Department of General, Oncological and Endocrinological Surgery, Regional Hospital, Kielce, Poland ${ }^{9}$ Department of Gastrointestinal Surgery, Medical University of Silesia, Katowice, Poland ${ }^{10}$ Department of Digestive Tract Diseases, Medical University of Lodz, Lodz, Poland ${ }^{11}$ Department of Gastroenterology and Hepatology, School of Medicine, Medical University of Silesia, Katowice, Poland ${ }^{12}$ Department of Gastroenterology, Medical University of Lublin, Lublin, Poland ${ }^{13}$ Department of Gastroenterology and Internal Medicine, Medical University of Bialystok, Bialystok, Poland ${ }^{14}$ Department of the Prevention of Alimentary Tract Diseases, Faculty of Medicine and Health Science, Jan Kochanowski University, Kielce, Poland

Key words: acute pancreatitis, management, treatment.

Address for correspondence: Prof. Grażyna Rydzewska MD, PhD, Department of Internal Medicine and Gastroenterology with IBD Subdivision, Central Clinical Hospital of the Ministry of the Interior, 137 Woloska St, 02-507 Warsaw, Poland, e-mail: grazyna.rydzewska@cskmswia.pl

\begin{abstract}
The presented recommendations concern the current management of acute pancreatitis. The recommendations relate to the diagnostics and treatment of early and late phases of acute pancreatitis and complications of the disease taking into consideration surgical and endoscopic methods. All the recommendations were subjected to voting by the members of the Working Group of the Polish Pancreatic Club, who evaluated them every single time on a five-point scale, where A means full acceptance, $B$ means acceptance with a certain reservation, $C$ means acceptance with a serious reservation, $D$ means rejection with a certain reservation and $\mathrm{E}$ means full rejection. The results of the vote, together with commentary, are provided for each recommendation.
\end{abstract}

Acute pancreatitis (AP) is an inflammatory state of the organ, clinically characterized by acute abdominal pain with a concomitant increase in the activity of serum pancreatic enzymes. The incidence is approximately 72 cases $/ 100000$ inhabitants per year [1], but an increasing trend is observed. Significant progress in accu- rate diagnostics and proper treatment has contributed to a significant reduction in the case fatality rate, especially in the course of the severe form of the disease. In the course of AP during the first 2 weeks deaths are mainly due to generalized systemic inflammatory response syndrome (SIRS) and multiple organ dysfunction syndrome. 
In the later period, the case fatality rate is the result of infection of pancreatic necrosis, sepsis and its complications. The total case fatality rate in the course of AP is about $10 \%$, but in patients with the severe form of the disease it may be up to $30 \%$. Clinical management of AP has been significantly modified in recent years [2, 3]

\section{Identification of AP should be based on the pres-} ence of two of the three following criteria:

a) abdominal pain typical for AP,

b) more than a three-fold increase in amylase and lipase activity,

c) abnormalities in imaging studies characteristic of AP.

$A-86 \%, B-14 \%, C-0 \%, D-0 \%, E-0 \%$.

The complaint most frequently reported by patients is acute abdominal pain, located mainly in the middle epigastrium or radiating to the lumbar region. This symptom is usually accompanied by flatulence, nausea and vomiting. A biochemical criterion of identification of AP is increased serum amylase and lipase activity [4]. The diagnosis should not be based on determination of the activity of only one enzyme due to an ambiguous sensitivity and specificity as well as a negative and positive predictive value of a single parameter $[5,6]$. The activity of the above enzymes as well as pain intensity does not correlate with the severity of the course of AP. In diagnostically ambiguous cases it is recommended to perform a computed tomography scan (CT) or magnetic resonance imaging (MRI) of the abdomen. An ultrasound examination generally does not have diagnostic value in the first days of pancreatitis, but it should be performed in the context of differential diagnostics and/or determination of a possible aetiology of AP.

In accordance with the revision of the Atlanta classification it is recommended to determine the beginning of AP as the time of onset of abdominal pain, and also the length of time between that moment and the hospital admission.

\section{The most common aetiological factors of AP are} cholelithiasis and alcohol. In other cases less common causes should be considered, including hypertriglyceridaemia.

A - $100 \%, B-0 \%, C-0 \%, D-0 \%, E-0 \%$.

Cholelithiasis constitutes approximately $35-45 \%$ of the causes of AP. However, it should be noted that only $3-7 \%$ of patients with cholelithiasis would develop AP. After elimination of an alcoholic cause as the second possible factor of AP, hypertriglyceridaemia should be taken into account as the third possible cause. It is believed that a triglyceride level above $1000 \mathrm{mg} / \mathrm{dl}$ can be a causal factor of AP. Other causes, including drug-induced, post-traumatic and hereditary, are significantly more frequent. Genetic testing (PRSS1, CFTR, SPINK1 gene mutations) may be considered in young patients with a family history of recurrent AP of unknown cause, after elimination of the fundamental causes. When determining the aetiology of AP in patients over the age of 40 , the possibility of pancreatic cancer must also be taken into account.

\section{Patients with AP of unidentified aetiology (idio- pathic inflammation) should be referred to refer- ence centres for further diagnostics. \\ A - $86 \%, B-14 \%, C-0 \%, D-0 \%, E-0 \%$.}

Patients with idiopathic pancreatitis or those in whom the cause of pancreatitis has not been established should be subjected to elective endoscopic ultrasound (EUS) immediately after the symptoms have subsided. It should be remembered that in over $50 \%$ of cases of so-called "idiopathic" AP we can identify the biliary cause of the disease - the so-called biliary sludge (microlithiasis) [7]. A diagnostic method that enables one to reliably assess the lesions in the gallbladder, not always visible in the classic ultrasound examination, is EUS [8]. This is particularly important in the aspect of prophylaxis of recurrent pancreatitis of unclear aetiology.

As it was previously emphasized, young patients with recurrences of AP of unclear aetiology should be referred for genetic testing. Autoimmune causes, pancreatic cancer or a tumour of the major duodenal papilla must also be excluded.

\section{Computed tomography of the abdomen with con- trast (CT) or magnetic resonance imaging of the abdomen (MRI) should be performed in patients with predicted severe or moderate $A P$, whose condition does not improve within 48-72 h, or the diagnosis is uncertain. \\ A - 86\%, B - $14 \%$, C - 0\%, D - 0\%, E - 0\%.}

Ultrasound examination of the abdomen should be performed in all the patients with AP. It allows diagnosis of a possible biliary cause of AP with clinically acceptable accuracy. Selection of a method of imaging of the abdomen should be appropriate for the patient's clinical condition. Performing a CT or MRI scan of the 
abdomen in every patient with AP, especially in patients with predicted mild form of the disease, seems to be groundless. Both these imaging methods seem to be equivalent in the early assessment of the staging of local complications and possible necrosis in the course of AP $[9,10]$. Magnetic resonance imaging with an option to visualize the biliary tracts and pancreatic duct (MRCP) has an advantage in the diagnostics of possible choledocholithiasis or damage to the main pancreatic duct. Magnetic resonance is sometimes useful in patients who are allergic to intravenous iodine contrast agents.

5. In accordance with the revision of the Atlanta classification, a three-point scale of severity of AP (mild, moderate and severe) is recommended. Predicting the severity of AP should be done using scales taking into account the clinical condition of the patient, laboratory tests and imaging studies. The scales recommended for predicting the severity of AP are: the BISAP scale, APACHE II or APACHE II-O scale, depending on the experience of the centre.

A - 93\%, B - 0\%, C - 0\%, D - 7\%, E- 0\%.

Determination of the severity of AP in the early phase of the disease mainly depends on the presence and duration of multiple organ dysfunction syndrome (transient or persistent), diagnosed according to the modified Marshall scale. In accordance with the revision of the Atlanta classification, AP is divided into: a mild form, characterized by lack of presence of organ dysfunction and local or systemic complications; a moderate form, characterized by transient $(<48 \mathrm{~h})$ organ dysfunction and/or the presence of local complications; and a severe form, characterized by persistent (> $48 \mathrm{~h}$ ) organ dysfunction concerning one or several organs [11]. The scales recommended for predicting the severity of AP are the APACHE II-O and the BISAP scales [12-15]. The individual biochemical exponents, both those indicating activation of the inflammatory process (e.g. C-reactive protein (CRP), IL-2, IL-6, IL-8, procalcitonin) and those indicating complications in other organs (eGFR, NGAL, creatinine, suPAR, haematocrit) may be useful in the preliminary assessment of the patient's condition [16-21]. Among others, concentrations of D-dimer, CRP and procalcitonin are helpful in predicting the severity of $\mathrm{AP}$ in subsequent days $[22,23]$. An accurate imaging examination of abdominal cavity, i.e. CT or MRI, allows reliable assessment of the progress of lesions at a later stage of the disease (> $72 \mathrm{~h}$ ).
6. Assessment of hemodynamic status is a fundamental action which must be done as soon as possible after the patient's admission to the hospital. Patients with multiple organ dysfunction should be treated in departments where intensive monitoring of vital signs is possible. $A-100 \%, B-0 \%, C-0 \%, D-0 \%, E-0 \%$.

In patients with the severe form of AP, monitoring of vital signs and supporting the cardiovascular system, lungs, kidneys and liver function may reduce the adverse systemic consequences. Especially in the early days of the disease, the patients should be hospitalized in departments that ensure the monitoring of vital signs. Treatment in an intensive care unit should be considered if the APACHE II scale score was $\geq 8$ points or the APACHE-O scale score was $\geq 10$ points on the first day of admission to the hospital, persistent ( $>48 \mathrm{~h}$ ) systemic inflammatory response syndrome is present as well as elevated ( $>44 \%$ ) haematocrit level, creatinine concentration is elevated ( $>1.8 \mathrm{mg} / \mathrm{dl}$ ), there are concomitant cardiovascular system diseases, respiratory system diseases, or obesity, and the patient's age is over 60 years.

\section{Basic therapeutic management in the initial phase of AP includes intensive intravenous hy- dration and pain management. \\ A - $100 \%, B-0 \%, C-0 \%, D-0 \%, E-0 \%$.}

Intensive fluid therapy offers most advantege if started < $24 \mathrm{~h}$ from onset of pain. Intravenous hydration should be started at 5-10 ml/kg/h for 30-60 min (in severe volume depletion, even $20 \mathrm{ml} / \mathrm{kg} / \mathrm{h}$ ) and should be continued at $3 \mathrm{ml} / \mathrm{kg} / \mathrm{h}$ for up to $48 \mathrm{~h}$. However, there are data suggesting that aggressive hydration can increase multiorgan failure, particularly respiratory complications. The results of newer studies on the optimal hydration of patients with AP suggest that the volume of fluids to be administered during the first $24 \mathrm{~h}$ is 4.0-5.0 I. A very important aspect is control of the administered fluid therapy, which includes the assessment of fluid balance with special reference to diuresis (optimally $0.5-1 \mathrm{ml} / \mathrm{kg} / \mathrm{h}$ ), and assessment of the dynamics of changes in the value of blood urea nitrogen (BUN) concentration [24].

Currently, lactated Ringer's solution is considered as a preferred crystalloid, although the results of the tests are not conclusive [25]. A proper fluid therapy can reduce pain resulting from ischaemia of the abdominal organs in the course of AP and the resulting lactic acidosis. Pain treatment is the management reducing the signs and symptoms of shock. In analgesic treat- 
ment the choice of the preparation should be carried out in accordance with the analgesic ladder, and in the case of aggravated symptoms it is recommended to use narcotic analgesics. There is no clinical evidence that morphine intensifies the severity of course of AP. In moderate pain it is also possible to use non-steroidal anti-inflammatory drugs, paracetamol or weak opioid drugs, for example tramadol.

8. An endoscopic revision of biliary tracts should be carried out within $24 \mathrm{~h}$ in patients with symptoms of AP and concomitant acute cholangitis. A revision should also be considered every time in the course of predicted severe acute biliary pancreatitis without symptoms of cholangitis.

$A-100 \%, B-0 \%, C-0 \%, D-0 \%, E-0 \%$.

Some studies indicate that if the endoscopic revision of the biliary tracts is performed early, it can reduce the severity of the course of AP [26]. The indications for the performance of endoscopic retrograde cholangiopancreatography (ERCP) in the course of AP are: visible concrements in the main bile duct, main bile duct dilatation and a significant increase in the activity of liver enzymes persisting for 24-48 h without concomitant acute cholangitis. In the light of ambiguous results of clinical studies, urgent performance of endoscopic revision of biliary tracts, even in the clinical conditions discussed above, is controversial. Conclusions from the available studies suggest a benefit concerning a possible reduction in the number of complications, without any impact on the case fatality rate, only in severe acute biliary pancreatitis [27]. In the light of these reports, the performance of ERCP can be considered in the case of severe acute biliary pancreatitis. However, an absolute indication for urgent ERCP procedure is the presence of symptoms of acute cholangitis.

If an obstruction in the biliary tracts (e.g. concrements) is not present, the ERCP procedure is not necessary. In the course of establishing the biliary background of the disease, when the biochemical and/or clinical signs of cholestasis are absent, accurate diagnostic imaging of biliary tracts should be carried out in the form of endoscopic ultrasonography or magnetic resonance imaging of the biliary tracts. The above diagnostics is also indicated in pregnant patients as well as in the case of the expected altered anatomy of the biliary tracts, for example as a result of a previous surgical treatment. In the assessment of microlithiasis as a potential cause of AP, EUS seems to have an advantage over MRCP $[8,28]$. Recurrent AP of unknown cause - in spite of the appropriate diagnostics - is an indication for endoscopic revision of the biliary tracts. ERCP should not be indicated as a diagnostic method in any case of AP.

\section{Routine use of prophylactic antibiotic therapy in the course of severe AP is not recommended. $A-93 \%, B-7 \%, C-0 \%, D-0 \%, E-0 \%$.}

Antibiotic therapy should be applied during the treatment of concomitant infections, i.e. acute cholangitis, urinary tract infections, pneumonia, bacteraemia or catheter-related infections. These kinds of non-pancreatic infections are present in approximately $20 \%$ of patients with AP [29] and significantly increase the case fatality rate in the course of the disease [30]. Prophylactic use of antibiotic therapy in the prevention of infection of sterile pancreatic necrosis, occurring in approximately $30 \%$ of cases, is also not recommended. No relationship between the extent of necrosis and the possible risk of infection was observed [31]. Infection of necrosis should be considered if the clinical condition has not improved despite 7-14 days of treatment. It is best to use targeted antibiotic therapy. The treatment can be based on the results of culture of the puncture of the pancreatic necrosis, or wide-spectrum antibiotics with good penetration into the pancreatic tissue can possibly be used. Monobacterial infections with bacterial strains of intestinal origin, i.e.. Escherichia coli, Pseudomonas, Klebsiella or Enterococcus, constitute approximately $75 \%$ of cases. Therefore in the case of an empirical antibiotic therapy carbapenems seem to be the most effective group of antibiotics [32]. Concomitant routine anti-fungal therapy is not recommended [33]. Intestinal decontamination may have an impact on reducing the incidence of infections in the course of severe AP. There are reports on using rifaximin in decontamination, which seems to be an interesting proposal, mainly because of the lack of absorption of this eubiotic, although the usefulness of this method requires further research.

10. In the case of mild AP, oral feeding should be started immediately after the acute symptoms, i.e. abdominal pain, nausea and vomiting, have subsided. In the case of predicted severe AP, early enteral feeding is recommended. Parenteral nutrition is recommended only in case of intolerance of enteral feeding or in addition to enteral feeding in case of inability to maintain the appropriate caloric balance.

$A-92 \%, B-0 \%, C-8 \%, D-0 \%, E-0 \%$. 
Many studies confirm the positive impact of early enteral feeding on the course of severe AP [34-36]. Parenteral nutrition can be considered in case of very poor tolerance of enteral feeding, preventing an adequate energy supply, or extended persistence of paralytic ileus [37]. According to studies, enteral feeding, via both an enteral tube and a gastric tube, demonstrated comparable efficacy and safety of use, although in the case of patients with paralytic ileus and retention of gastric content gastric feeding generally is not well tolerated [38]. One of the latest studies on enteral feeding did not show a positive effect of early enteral feeding on the course of severe AP, not only compared to parenteral nutrition, but also compared to the standard oral diet [39]. This study has raised some doubts of the experts, although it should be mentioned that in the studied group in a significant percentage of patients with AP that had previously been predicted to be severe, the course of the disease turned out to be mild, which undoubtedly influenced such optimistic results. At the current stage it seems that this work does not invalidate many of the earlier reports and cannot become the basis for changes to the standard procedure, although it undoubtedly indicates that more research is needed on this topic.

11. Local complications of AP include acute peripancreatic fluid collections, pancreatic pseudocysts, acute necrotic collections and walled-off necrosis.

A - 92\%, B - 0\%, C - 8\%, D - 0\%, E- 0\%.

Asymptomatic fluid collections do not require any procedures [40, 41]. Infection of necrosis should be taken into account in patients whose clinical condition does not improve within 7-10 days of treatment or, despite the initial improvement, is rapidly deteriorating in the later stage of the disease, with concomitant fever and increase in leukocytosis. The infected necrosis is confirmed based on fine-needle aspiration biopsy under CT control with subsequent targeted antibiotic therapy. An acceptable procedure is the implementation of empirical antibiotic therapy. In the case of infected necrosis, the treatment procedures, preceded by antibiotic therapy, should be postponed for a few weeks (4-6) or for as long as the patient's condition remains stable. If the patient's clinical condition deteriorates, a procedural intervention should be considered. It seems that the minimally invasive procedures of removal of pancreatic necrosis have advantages over a surgical intervention, but the choice of intervention method should also depend on the experience of the centre and should be made individually depending on the conditions and technical possibilities [42, 43].
12. Other supporting methods of treatment of severe AP such as digestive decontamination, pentoxifylline, low-molecular-weight heparin or continuous regional arterial infusion (CRAI) of protease inhibitors and antibiotic infused intrapancreatically are not recommended, though they may be applied in accordance with the experience of the centre.

A - $84 \%, B-8 \%, C-8 \%, D-0 \%, E-0 \%$.

The data from the literature concerning treatment with protease inhibitors are not unambiguous. Most studies include the use of nafamostat, but it should be noted that most of the patients in the studied groups received in parallel local-intra-arterial or systemic antibiotic therapy, which could affect the test results. The conclusions from the randomized studies suggest positive clinical implications of the management described above [44, 45], and this management is also mentioned in Japanese recommendations. However, the available meta-analyses do not confirm a significant favourable impact of the use of protease inhibitors on the course of AP [46]. In the case of the other methods that were mentioned, the treatment should be individualized depending on the patient's clinical condition.

13. Prevention of AP after endoscopic retrograde cholangiopancreatography should include the use of diclofenac and adequate hydration of the patient.

A - $100 \%, B-0 \%, C-0 \%, D-0 \%, E-0 \%$.

Current pharmacological recommendations include rectal administration of diclofenac or indomethacin immediately before or immediately after the procedure, especially in the group of patients at high risk of AP after ERCP; however, the results of the studies are not unambiguous [47, 48]. Aggressive fluid therapy probably reduces the risk of AP after ERCP [49].

14. In the case of biliary AP, cholecystectomy should always be performed in the mild form and is recommended as soon as possible after the AP, preferably during the same hospitalization. In the severe form, postponement of surgery appears to be justified until the resolution of inflammatory infiltrates and regression of intra-abdominal fluid collections.

A - $92 \%, B-8 \%, C-0 \%, D-0 \%, E-0 \%$. 
Abandoning cholecystectomy is associated with approximately $30 \%$ risk of recurrence of acute pancreatitis, during 6-18 weeks, and the risk of acute cholecystitis. The risk of recurrence of AP is particularly high in patients who did not have endoscopic sphincterotomy performed [50]. If the possibility of the presence of concrements in the common bile duct is high, endoscopic sphincterotomy before cholecystectomy should not raise doubts. When there is a low degree of probability of concrements in the common bile duct, intraoperative cholangiography during cholecystectomy should be the method of choice for final exclusion/confirmation of any possible irregularities. In the case of a moderate degree of probability of the presence of concrements, MRCP or EUS should be performed prior to cholecystectomy, and in the event of confirmation of the presence of concrements, preoperative sphincterotomy is indicated. Moreover, cholecystectomy performed in the week after endoscopic sphincterotomy significantly reduces the risk of conversion of laparoscopic cholecystectomy to the classic cholecystectomy, perioperative morbidity and the length of hospital stay [51]. Endoscopic sphincterotomy without subsequent cholecystectomy may be an alternative treatment procedure in patients with contraindications to surgical treatment [52]. The use of ursodeoxycholic acid can be an alternative method of preventing the recurrences of AP when microlithiasis is suspected, especially in patients with contraindications to surgery [53]. This seems particularly interesting in the aspect of additional reports, indicating a protective effect of ursodeoxycholic acid in the case of acute biliary pancreatitis [54].

15. The detection of asymptomatic acute peripancreatic fluid collections, acute necrotic collections and walled-off necrosis is not an indication for endoscopic or surgical intervention, regardless of the size and location of the lesions. In the case of symptomatic local complications, the preferred route of intervention is the minimally invasive way (endoscopic or laparoscopic).

A - $100 \%, B-0 \%, C-0 \%, D-0 \%, E-0 \%$.

Asymptomatic local complications are not indications for an early intervention. Even after 6 weeks the asymptomatic pancreatic pseudocysts do not constitute grounds for an intervention, regardless of their size [2].

In minimally invasive management, percutaneous drainage, laparoscopic drainage or gastric endoscopic drainage should be considered, depending on the experience of the centre. At present, there are not enough data to recommend endoscopic drainage as a management of choice [55].

16. The detection of infected pancreatic necrosis is not an absolute indication for surgical treatment. Decisions concerning an open necrosectomy should be taken individually, after having exhausted the possibilities of conservative or minimally invasive treatment.

A $-92 \%, B-8 \%, C-0 \%, D-0 \%, E-0 \%$.

As discussed previously, currently the preferred management in treatment of severe AP is an expectant management. Aseptic necrosis is not an indication for an intervention or antibiotic prophylaxis, while in the case of suspected infected necrosis a conservative management, including the antibiotic therapy mentioned before, is most appropriate. In the case of deteriorating clinical condition of the patient, despite the properly applied conservative management, the indications for surgical intervention should be limited and considered individually in each case [2, 31, 32].

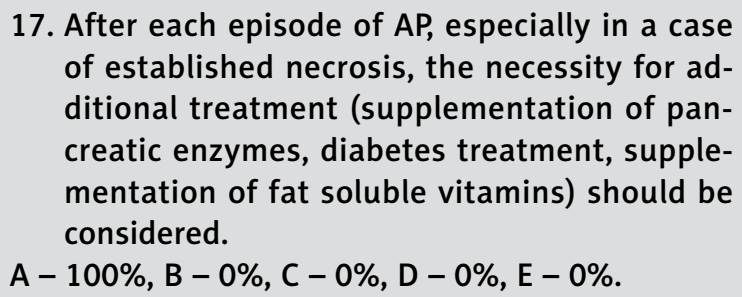

In the case of patients who have experienced severe necrotic $A P$, it should be remembered that disorders of glycaemic control, including the possibility of developing diabetes, are possible. In these patients the risk of diabetes during the 5 -year follow-up period is more than twice as high as it was before the occurrence of AP [56]. Similarly, the exocrine pancreatic function should be monitored and the supplementation of pancreatic enzymes and fat soluble vitamins should be introduced at the appropriate moment. The phrase restitutio ad integrum - which means complete regeneration of the pancreas and was often mentioned in previous years - concerns mainly mild oedematous forms of pancreatitis.

\section{Conclusions}

Guidelines concerning the management of AP generally are in accordance with the recommendations of the IAP (International Association of Pancreatology) and contain the indications based on the available lit- 
erature that have been discussed by the experts and in general do not raise doubts. They are based on the new Atlanta classification and also draw much more attention than previously to the aggressive conservative treatment in severe forms and expectant management in case of a need for surgical intervention. These guidelines, of course, do not exempt the specialist from tracking the new multicentre randomized trials (http://apps.who. int/trialsearch/), the results of which can sometimes specify or change the current recommendations.

\section{References}

1. Koziel D, Gluszek S. Epidemiologia ostrego zapalenia trzustki w Polsce - wybrane problemy. Medical Studies/Studia Medyczne 2016; 32: 1-3.

2. Working Group IAPAPAAPG. IAP/APA evidence-based guidelines for the management of acute pancreatitis. Pancreatology 2013; 13: e1-15.

3. Tenner S, Baillie J, DeWitt J, et al. American College of Gastroenterology guideline: management of acute pancreatitis. Am J Gastroenterol 2013; 108: 1400-16.

4. Winslet M, Hall C, London NJ, Neoptolemos JP. Relation of diagnostic serum amylase levels to aetiology and severity of acute pancreatitis. Gut 1992; 33: 982-6.

5. Shah AM, Eddi R, Kothari ST, et al. Acute pancreatitis with normal serum lipase: a case series. JOP 2010; 11: 369-72.

6. Clavien PA, Robert J, Meyer P, et al. Acute pancreatitis and normoamylasemia. Not an uncommon combination. Ann Surg 1989; 210: 614-20.

7. Lee SP, Nicholls JF, Park HZ. Biliary sludge as a cause of acute pancreatitis. N Engl J Med 1992; 326: 589-93.

8. Zidi SH, Prat F, Le Guen O, et al. Use of magnetic resonance cholangiography in the diagnosis of choledocholithiasis: prospective comparison with a reference imaging method. Gut 1999; 44: 118-22.

9. Arvanitakis M, Delhaye M, De Maertelaere V, et al. Computed tomography and magnetic resonance imaging in the assessment of acute pancreatitis. Gastroenterology 2004; 126: 715-23.

10. Stimac D, Miletic D, Radic M, et al. The role of nonenhanced magnetic resonance imaging in the early assessment of acute pancreatitis. Am J Gastroenterol 2007; 102: 997-1004.

11. Bollen TL, Singh VK, Maurer R, et al. A comparative evaluation of radiologic and clinical scoring systems in the early prediction of severity in acute pancreatitis. Am J Gastroenterol 2012; 107: 612-9.

12. Dominguez-Munoz JE, Carballo F, Garcia MJ, et al. Evaluation of the clinical usefulness of APACHE II and SAPS systems in the initial prognostic classification of acute pancreatitis: a multicenter study. Pancreas 1993; 8: 682-6.

13. Wu BU, Johannes RS, Sun X, et al. The early prediction of mortality in acute pancreatitis: a large population-based study. Gut 2008; 57: 1698-703.

14. Johnson CD, Toh SK, Campbell MJ. Combination of APACHE-II score and an obesity score (APACHE-O) for the prediction of severe acute pancreatitis. Pancreatology 2004; 4: 1-6.
15. Papachristou GI, Muddana V, Yadav D, et al. Comparison of BISAP, Ranson's, APACHE-II, and CTSI scores in predicting organ failure, complications, and mortality in acute pancreatitis. Am J Gastroenterol 2010; 105: 435-41; quiz 42.

16. Muddana V, Whitcomb DC, Khalid A, et al. Elevated serum creatinine as a marker of pancreatic necrosis in acute pancreatitis. Am J Gastroenterol 2009; 104: 164-70.

17. Brown A, Orav J, Banks PA. Hemoconcentration is an early marker for organ failure and necrotizing pancreatitis. Pancreas 2000; 20: 367-72.

18. Lipinski M, Rydzewska-Rosolowska A, Rydzewski A, Rydzewska G. Urinary neutrophil gelatinase-associated lipocalin as an early predictor of disease severity and mortality in acute pancreatitis. Pancreas 2015; 44: 448-52.

19. Papachristou GI, Whitcomb DC. Predictors of severity and necrosis in acute pancreatitis. Gastroenterol Clin North Am 2004; 33: 871-90.

20. Rosołowski M, Wereszczyńska-Siemiątkowska U, Mroczko B, Dąbrowski A. Interleukin 2 and its soluble receptor - important predictors of severity and mortality in human acute pancreatitis. Gut 2008; 57: A400.

21. Lipinski M, Rydzewska-Rosolowska A, Rydzewski A, Rydzewska G. Soluble urokinase-type plasminogen activator receptor (suPAR) in patients with acute pancreatitis; progress or 'suPAR' revolution in predicting acute pancreatitis outcome. Gastroenterology 2015; 148: S-679.

22. Kylanpaa-Back ML, Takala A, Kemppainen E, et al. Procalcitonin strip test in the early detection of severe acute pancreatitis. $\mathrm{Br}$ J Surg 2001; 88: 222-7.

23. Leese T, Shaw D, Holliday M. Prognostic markers in acute pancreatitis: can pancreatic necrosis be predicted? Ann R Coll Surg Engl 1988; 70: 227-32.

24. Aakash A, Manish M, Rakesh K. Fluid resuscitation in acute pancreatitis. World J Gastroenterol 2014; 20: 18092-103.

25. Lipinski M, Rydzewska-Rosolowska A, Rydzewski A, Rydzewska G. Fluid resuscitation in acute pancreatitis: normal saline or lactated Ringer's solution? World J Gastroenterol 2015; 21: 9367-72.

26. van Santvoort HC, Besselink MG, de Vries AC, et al. Early endoscopic retrograde cholangiopancreatography in predicted severe acute biliary pancreatitis: a prospective multicenter study. Ann Surg 2009; 250: 68-75.

27. Moretti A, Papi C, Aratari A, et al. Is early endoscopic retrograde cholangiopancreatography useful in the management of acute biliary pancreatitis? A meta-analysis of randomized controlled trials. Dig Liver Dis 2008; 40: 379-85.

28. Kohut M, Nowakowska-Dulawa E, Marek T, et al. Accuracy of linear endoscopic ultrasonography in the evaluation of patients with suspected common bile duct stones. Endoscopy 2002; 34: 299-303.

29. Besselink MG, van Santvoort HC, Boermeester MA, et al. Timing and impact of infections in acute pancreatitis. Br J Surg 2009; 96: 267-73.

30. Wu BU, Johannes RS, Kurtz S, Banks PA. The impact of hospital-acquired infection on outcome in acute pancreatitis. Gastroenterology 2008; 135: 816-20.

31. Bradley EL 3rd, Allen K. A prospective longitudinal study of observation versus surgical intervention in the management of necrotizing pancreatitis. Am J Surg 1991; 161: 19-24. 
32. Manes G, Uomo I, Menchise A, et al. Timing of antibiotic prophylaxis in acute pancreatitis: a controlled randomized study with meropenem. Am J Gastroenterol 2006; 101: 1348-53.

33. Trikudanathan G, Navaneethan U, Vege SS. Intra-abdominal fungal infections complicating acute pancreatitis: a review. Am J Gastroenterol 2011; 106: 1188-92.

34. McClave SA, Chang WK, Dhaliwal R, Heyland DK. Nutrition support in acute pancreatitis: a systematic review of the literature. JPEN J Parenter Enteral Nutr 2006; 30: 143-56.

35. Al-Omran M, Albalawi ZH, Tashkandi MF, Al-Ansary LA. Enteral versus parenteral nutrition for acute pancreatitis. Cochrane Database Syst Rev 2010; 10: CD002837.

36. Wereszczynska-Siemiatkowska U, Swidnicka-Siergiejko A, Siemiatkowski A, Dabrowski A. Early enteral nutrition is superior to delayed enteral nutrition for the prevention of infected necrosis and mortality in acute pancreatitis. Pancreas 2013; 42: 640-6.

37. Casaer MP, Mesotten D, Hermans G, et al. Early versus late parenteral nutrition in critically ill adults. N Engl J Med 2011; 365: 506-17.

38. Singh N, Sharma B, Sharma M, et al. Evaluation of early enteral feeding through nasogastric and nasojejunal tube in severe acute pancreatitis: a noninferiority randomized controlled trial. Pancreas 2012; 41: 153-9.

39. Bakker OJ, van Brunschot S, van Santvoort HC, et al. Early versus on-demand nasoenteric tube feeding in acute pancreatitis. N Engl J Med 2014; 371: 1983-93.

40. Walser EM, Nealon WH, Marroquin S, et al. Sterile fluid collections in acute pancreatitis: catheter drainage versus simple aspiration. Cardiovasc Intervent Radiol 2006; 29: 102-7.

41. Banks PA, Bollen TL, Dervenis C, et al. Classification of acute pancreatitis - 2012: revision of the Atlanta classification and definitions by international consensus. Gut 2013; 62: 102-11.

42. van Santvoort HC, Besselink MG, Bakker OJ, et al. A step-up approach or open necrosectomy for necrotizing pancreatitis. N Engl J Med 2010; 362: 1491-502.

43. Smoczynski M, Marek I, Dubowik M, et al. Endoscopic drainage/debridement of walled-off pancreatic necrosis: single center experience of 112 cases. Pancreatology 2014; 14: 137-42.

44. Piascik M, Rydzewska G, Milewski J, et al. The results of severe acute pancreatitis treatment with continuous regional arterial infusion of protease inhibitor and antibiotic: a randomized controlled study. Pancreas 2010; 39: 863-7.

45. Imaizumi H, Kida M, Nishimaki $\mathrm{H}$, et al. Efficacy of continuous regional arterial infusion of a protease inhibitor and antibiotic for severe acute pancreatitis in patients admitted to an intensive care unit. Pancreas 2004; 28: 369-73.

46. Seta T, Noguchi Y, Shimada T, et al. Treatment of acute pancreatitis with protease inhibitors: a meta-analysis. Eur J Gastroenterol Hepatol 2004; 16: 1287-93.

47. Ding $X$, Chen $M$, Huang $S$, et al. Nonsteroidal anti-inflammatory drugs for prevention of post-ERCP pancreatitis: a meta-analysis. Gastrointest Endosc 2012; 76: 1152-9.

48. Levenick JM, Gordon SR, Fadden LL, et al. Rectal indomethacin does not prevent post-ERCP pancreatitis in consecutive patients. Gastroenterology 2016; 150: 911-7.

49. Buxbaum J, Yan A, Yeh K, et al. Aggressive hydration with lactated Ringer's solution reduces pancreatitis after endoscop- ic retrograde cholangiopancreatography. Clin Gastroenterol Hepatol 2014; 12: 303-7e1.

50. Hernandez V, Pascual I, Almela P, et al. Recurrence of acute gallstone pancreatitis and relationship with cholecystectomy or endoscopic sphincterotomy. Am J Gastroenterol 2004; 99: 2417-23.

51. Schiphorst AH, Besselink MG, Boerma D, et al. Timing of cholecystectomy after endoscopic sphincterotomy for common bile duct stones. Surg Endosc 2008; 22: 2046-50.

52. Saito M, Tsuyuguchi T, Yamaguchi T, et al. Long-term outcome of endoscopic papillotomy for choledocholithiasis with cholecystolithiasis. Gastrointest Endosc 2000; 51: 540-5.

53. Saraswat VA, Sharma BC, Agarwal DK, et al. Biliary microlithiasis in patients with idiopathic acute pancreatitis and unexplained biliary pain: response to therapy. J Gastroenterol Hepatol 2004; 19: 1206-11.

54. Katona M, Hegyi P, Kui B, et al. A novel, protective role of ursodeoxycholate in bile-induced pancreatic ductal injury. Am J Physiol Gastrointest Liver Physiol 2016; 310: G193-204.

55. Babu RY, Gupta R, Kang M, et al. Predictors of surgery in patients with severe acute pancreatitis managed by the step-up approach. Ann Surg 2013; 257: 737-50.

56. Das SL, Singh PP, Phillips AR et al. Newly diagnosed diabetes mellitus after acute pancreatitis: a systematic review and meta-analysis. Gut 2014; 63: 818-31.

Received: 15.05 .2016

Accepted: 22.05.2016 